\title{
Capabilities of Digital Software for Creating a 3D Printed Retainer
}

\author{
Dobromira Shopova $^{1 *}$ (D), Miroslava Yordanova² ${ }^{\mathbb{D}}$, Svetlana Yordanova² \\ ${ }^{1}$ Department of Prosthetic Dentistry, Faculty of Dental Medicine, Medical University, Plovdiv, Bulgaria; ${ }^{2}$ Department of \\ Orthodontics, Faculty of Dental Medicine, Medical University, Plovdiv, Bulgaria
}

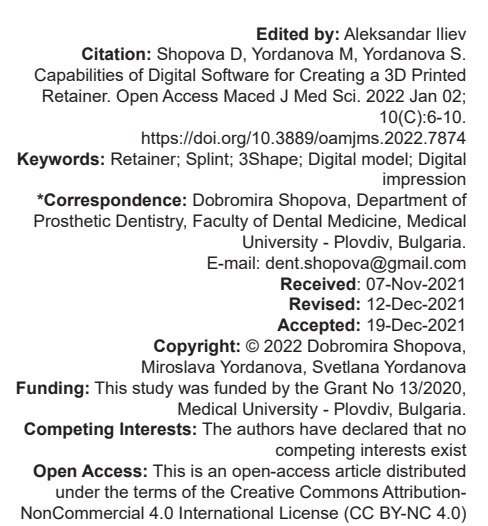

Abstract

INTRODUCTION: The retention phase of orthodontic treatment is an important phase to prevent teeth returning to their initial position. An innovative solution for creating a retainer is through $3 \mathrm{D}$ printing by digital design.

AIM: The purpose of this article is to demonstrate the capabilities of digital software in the creation of stabilization splint, type retainer, and after orthodontic treatment.

MATERIAL AND METHODS: An intraoral scan of an orthodontically treated patient (18 years) was performed. A Trios Color scanner (3Shape) was used. 3Shape Dental system design - splint studio was used for file processing and design creation. The method of 3D printing was applied to make the retainer. With this method, the objects are created layer by layer from melted material with different nature. It was made by Dental LT Clear Resin (a biocompatible material) with Formlabs Form 2 printer

RESULTS: Depending on the selected thickness of the retainer and the position of the lower jaw, the software generates different distances between the dentitions. The digital design allows a change of $0.1 \mathrm{~mm}$, which is impossible with a classic laboratory protocol. The resulting printed retainer fully meets our expectations - tooth adaptation, patient comfort, and aesthetics.

CONCLUSION: Digital software and design provide many opportunities for modern orthodontics.

\section{Introduction}

In modern dentistry, digitalization is indispensable part. 3D printing and CAD/CAM technologies are the two main methods for creating digitally designed objects. However, they are quite different in nature - additive manufacturing builds the object layer by layer, while CAD/CAM cuts it from a homogeneous block of material [1], [2]. Digital software reveals many possibilities for creating various structures - models, crowns, surgical guides, splints, and many more. The processing of the digital model is very precise and allows the production of fine details, which with the classical methods are difficult to achieve [3].

Properly, performed orthodontic treatment ends with a retention phase. Preservation of results is equally important [4]. The stable position of the teeth can be affected by the periodontal and gingival tissues, tongue, masticatory muscles, and occlusal forces, as well as the residual growth of the facial bones if the balance between them is disturbed [5], [6].

Retainers are devices used in the retention phase of orthodontic treatment. They aim to maintain the achieved result by opposing the bone and soft tissues in the newly achieved position. They are removable and fixed. The choice of the retainer's type depends on a number of factors - the type of orthodontic treatment, positioning of teeth from atypical places, available space for proper occlusion with fixed retainers, cariesresistant or non-resistant dentition, etc. [7], [8].

The most widely used removable retainer is the vacuum-formed retainer. Its advantages are: Easy technology, high aesthetics, and fast adaptation to speech [9], [10], [11]. Its disadvantages are: Relatively fast wear (about 1 year), easy deformation, tearing, and perforation [12], [13]. Acrylic retainers (Hawley retainers) are more durable [14], [15]. However, their production has a complicated laboratory protocol. In cases of untimely fabrication or delay in the laboratory, partial return of the teeth to an undesirable position is possible. Fixed retainers are more comfortable for patients due to the lack of care for them, except for hygienic habits, and some restrictions in the type of food (nuts, chewing gum, and solid foods). However, there is a possibility of detachment and fractures [16].

The application of a digital work protocol requires the introduction of modern methods such as intraoral scanning. Intraoral scanning of the dentition eliminates the need to take classic impressions from the patient's mouth. The created virtual models allow working on them 
and making the desired structure without casting gypsum models. The obtained constructions are very accurate, because a series of stages are omitted, each of which includes an error - shrinkage of the impression material, expansion of gypsum, shrinkage of the acrylic resin during polymerization, etc. [17]. The material for 3D printed retainers is a transparent biocompatible resin Class II with high durability, but still printed retainers have not been sufficiently studied for orthodontic purposes [18], [19].

This encourages orthodontists to look for options that combine the positive qualities - aesthetics, function, durability, and easy work protocol.

The purpose of this article is to demonstrate the capabilities of digital software in the creation of stabilization splint, type retainer, and after orthodontic treatment.

\section{Materials and Methods}

An intraoral scan of an orthodontically treated patient (18-years-old) was performed. A Trios Color scanner (3Shape) was used. When entering the patient information, the established sequence, and the "splint" option for creating, the order was observed. The upper jaw was marked as supporting the splint. The scan started from the lower jaw (antagonists), followed by the upper jaw and bilaterally in the bite. The created digital images were sent as an STL file by e-mail to the laboratory.

3Shape Dental system design - splint studio was used for file processing and creation of the retainer design.

The method of 3D printing was applied to make the retainer. Formlabs Form 2 printer was used, with biocompatible resin Dental LT Clear Resin.

\section{Results}

After the starting of 3Shape Dental system design - splint studio, various design possibilities open up. This includes:

$>\quad$ Different thickness of the splint vestibularly and occlusally,

$>\quad$ Ability to move the lower jaw back and forward (protrusion and retrusion),

Different drawing the outlines depending on the retention sections and the function of the splint,

$>\quad$ Different relief of the occlusal surface - in occlusion with the antagonists, without relief, and with an inclined surface to the antagonists in certain areas.

When entering the required thickness for the retainer $0.7 \mathrm{~mm}$, the program automatically generates an opening of $3.0 \mathrm{~mm}$. This is due to the fact that the two jaws do not open parallel to each other. When opening $0.7 \mathrm{~mm}$ at the extreme distal point, the dental arches move away by $3.0 \mathrm{~mm}$ in the area of the frontal teeth, Figure 1.

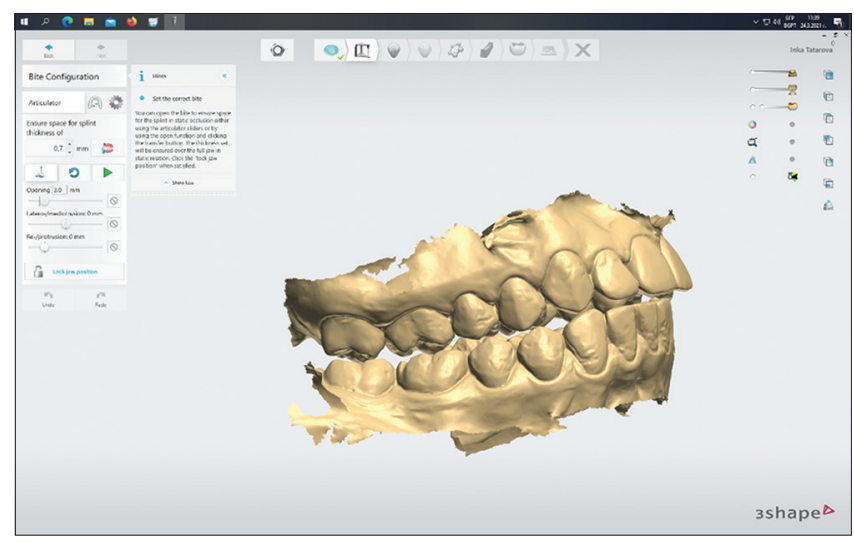

Figure 1: Position of the upper and lower jaw with $3 \mathrm{~mm}$ opening due to $0.7 \mathrm{~mm}$ thickness of the splint in the last molars area

This thickness is normal for classical bruxism splints but is not appropriate for retainers. Therefore, other options for creating the retainer by digital design were sought, Table 1:

Table 1: Relation between thickness, opening, and protrusion according to the 3 Shape Dental system design - splint studio

\begin{tabular}{llllllllllllll}
\hline Thickness & 0.6 & 0.6 & 0.6 & 0.6 & 0.6 & 0.6 & 0.7 & 0.7 & 0.7 & 0.7 & 0.7 & 0.7 & 0.7
\end{tabular} $\begin{array}{llllllllllllll}\text { Opening } & 1.3 & 1.2 & 1 & 0.8 & 0.7 & 0.7 & 1.1 & 0.9 & 0.8 & 1.2 & 1.5 & 1.9 & 3.0\end{array}$ \begin{tabular}{llllllllllllll} 
Protrusion & 0.3 & 0.5 & 0.8 & 1 & 1.2 & 1.4 & 1 & 1.2 & 1.4 & 0.8 & 0.5 & 0.3 & 0.0 \\
\hline
\end{tabular}

The main requirements for the final product we are looking for were:

> Optimal thickness vestubularly for wearing comfort during the day, which does not disturb the aesthetics and sound articulation,

Not more than $1 \mathrm{~mm}$ thick occlusally, which does not provoke changes in the joint and in intermaxillary relation

Slight protrusion of the lower jaw until the achievement of angle Class I in the area of molars and canines.

From the proposed options were selected: Thickness $0.6 \mathrm{~mm}$, opening $0.7 \mathrm{~mm}$, and protrusion $1.2 \mathrm{~mm}$.

The digital design allows a change of $0.1 \mathrm{~mm}$, which is impossible with a classic laboratory protocol. Setting the direction of placement of the retainer makes visible undercuts, which facilitates the selection of its boundaries and its positioning only in non-retention areas or unlocking the retention zones. Setting the same distance from the tooth surfaces ensures a stable position and an even retention effect. 
The retainer was designed in occlusion to the antagonists, which ensures the impossibility of the appearance of Godon's phenomenon of germination of single teeth or areas and non-traumatic treatment of the periodontium by single contact between the dentitions.

During the subsequent processing, it was made a balanced contact in the central occlusion and during lateral movements through the virtual articulator, Figures 2 and 3.

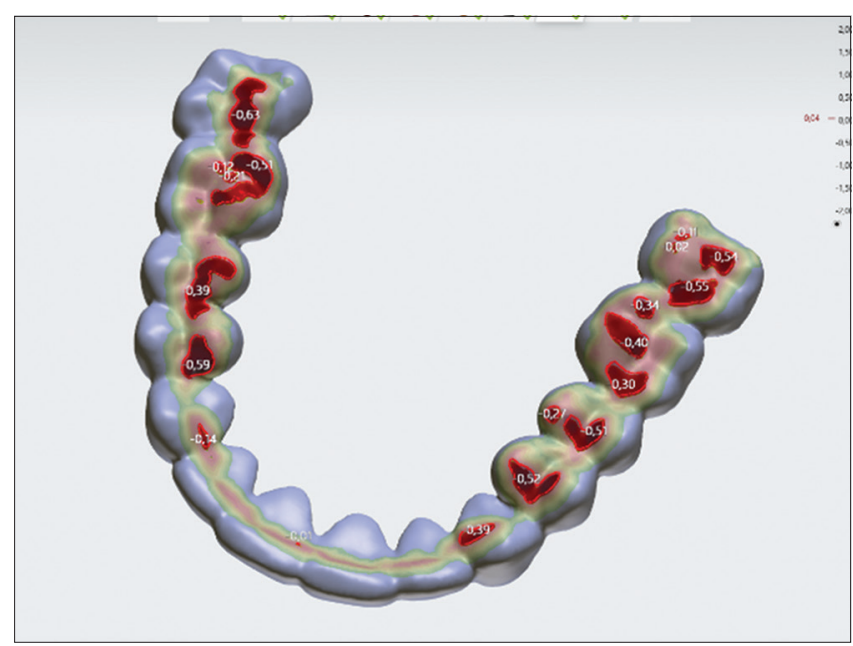

Figure 2: Balanced occlusion in the central position (3Shape Dental system design studio

To achieve this balance, digital software thickens the splint palatally. In this way, wider planar contacts are achieved, Figure 4.

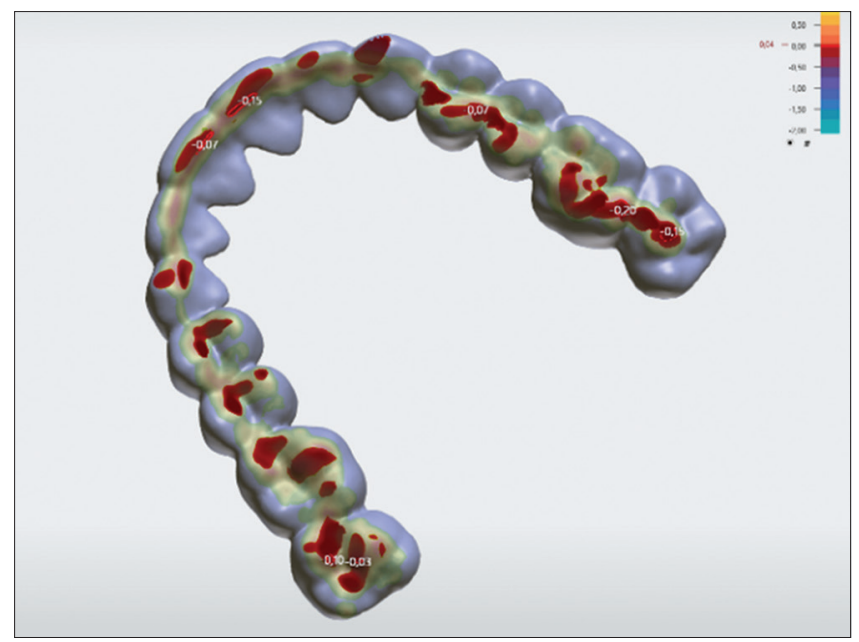

Figure 3: Balanced articulation contacts (3Shape Dental system design studio)

After completing the design, the retainer was made by $3 \mathrm{D}$ printing of biocompatible resin Dental LT Clear Resin. After minimal cleaning of the supports, it was placed in the patient's mouth. The patient reported a tight fit with uniform light pressure, which was also found clinically. No need to adjust the inner surfaces of the retainer, as well as occlusal. This was followed by oral and written instructions for usage, storage, hygiene, etc., Figures 5 and 6.

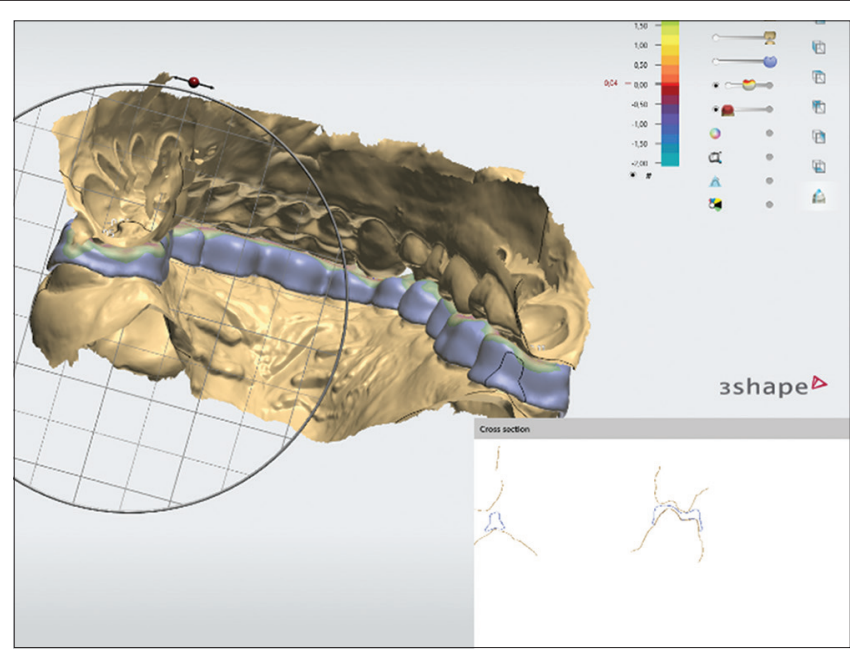

Figure 4: Palatal extension of the retainer

Discussion

For a retainer to be effective, it must be positioned tightly and passively on the tooth surfaces. The possible distance could provoke displacement of the teeth in the retention period. The digital design allows very precise setting of the minimum distance between the retainer and the tooth surfaces [3].

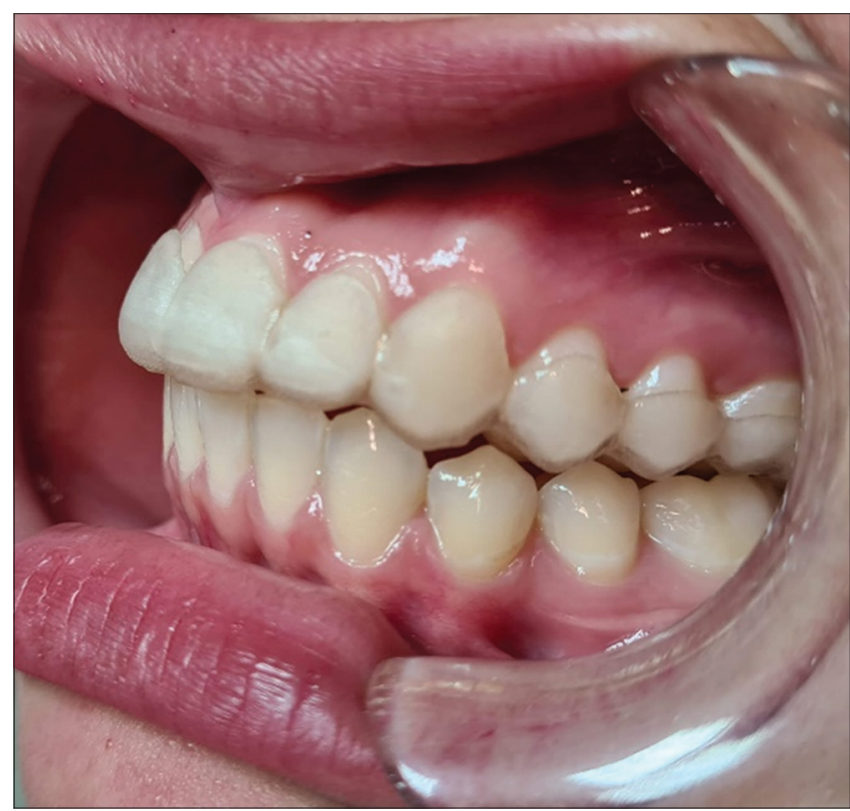

Figure 5: Vestibular view of the retainer

The thermoformed retainer due to its small thickness does not give many opportunities for adjustment and achieve even contacts. Although no data have been described on the need to achieve a balanced occlusion in the preparation of retainers [19], it is good to establish one due to the long-term required wearing period. In digital planning, it is possible to choose the type of occlusal surface - smooth, with a sloping surface, relief to antagonists, or a combination of all. The software also allows the production of a retainer with pre-adjusted surfaces [2]. 


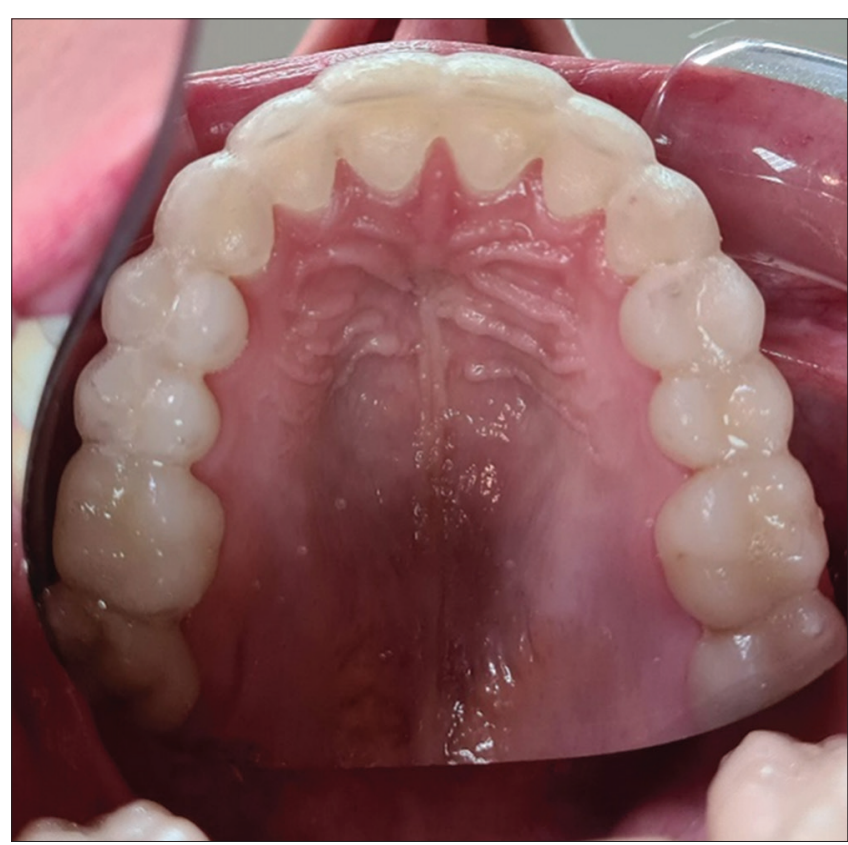

Figure 6: Palatal view of the retaine

A big advantage of the digital design is the quick reaction in case of breaking, forgetting, or repeating the retainer. The information stored in the database can be immediately forwarded to the printer and a product can be received within hours. This product can be an exact copy or make slight adjustments that have proved appropriate during the period of operation [16], [17], [18].

\section{Conclusion}

The final product obtained the parameters set in the digital design. Digital software offers many options for individual solutions in each clinical case. The accuracy of production by modern methods of 3D printing is incomparable with classical methods.

\section{Acknowledgments}

The design was performed at the CAD/CAM Center Dental Medicine at the Research Institute, Medical University - Plovdiv, Bulgaria.

\section{Author Contributions}

All authors have read and agreed to the published version of the manuscript.

\section{Institutional Review Board Statement}

The study was conducted according to the guidelines of the declaration of Helsinki, and approved by the Ethics Committee of Medical University of Plovdiv, Bulgaria - protocol №. 3/May 20, 2021.

\section{Informed Consent Statement}

Informed consent for participation in the study and publication of the data for research and educational purposes were obtained from the patient involved in the study.

\section{References}

1. Dawood A, Marti BM, Sauret-Jackson V, Darwood A. 3D printing in dentistry. Br Dent J. 2015;219(11):521-9. https://doi. org/10.1038/sj.bdj.2015.914

PMid:26657435

2. Mikolajczyk T, Malinowski T, Moldovan L, Fuwen $\mathrm{H}$, Paczkowski T, Ciobanu I. CAD CAM system for manufacturing innovative hybrid design using 3D printing. Proc Manufactur. 2019;32:22-8.

3. Zhou Q, Wang Z, Chen J, Song J, Chen L, Lu Y. Development and evaluation of a digital dental modeling method based on grating projection and reverse engineering software. J Prosthet Dent. 2016;115(1):42-6. https://doi.org/10.1016/j. prosdent.2015.06.016

PMid:26384536

4. Melrose C, Millett DT. Toward a perspective on orthodontic retention? Am J Orthod Dentofacial Orthop. 1998;113(5):507-14. https://doi.org/10.1016/s0889-5406(98)70261-6

PMid:9598608

5. Littlewood SJ, Millett DT, Doubleday B, Bearn DR, Worthington HV. Orthodontic retention: A systematic review. J Orthod 2006;33(3):205-12. https://doi. org/10.1179/146531205225021624

PMid:16926314

6. Joondeph DR. Stability, retention and relapse. In: Graber TM, Vanarsdall RL, Vig KW, editors. Orthodontics Current Principle and Techniques. $5^{\text {th }}$ ed. St. Louis: Mosby; 2012.

7. Johnston CD, Littlewood SJ. Retention in orthodontics. Br Dent J 2015;218:119-22. https://doi.org/10.1038/sj.bdj.2015.47 PMid:25686428

8. Available from: https://www.ddslab.com/orthodontics/retainerfinishing [Last accessed on 2021 Jul 29].

9. Sheridan JJ, LeDoux W, McMinn R. Essix retainers: Fabrication and supervision for permanent retention. $J$ Clin Orthod. 1993;27(1):37-45.

\section{PMid:8478438}

10. Saleh M, Hajeer MY, Muessig D. Acceptability comparison between Hawley retainers and vacuum-formed retainers in orthodontic adult patients: A single, randomized controlled trial. Eur J Orthod. 
2017;39(4):453-60. https://doi.org/10.1093/ejo/cjx024 PMid:28430890

11. Hichens $L$, Rowland $H$, Williams $A$, Hollinghurst $S$, Ewings $P$ Clark S. Costeffectiveness and patient satisfaction: Hawley and vacuum-formed retainers. Eur J Orthod. 2007;29(4):372-8. https://doi.org/10.1093/ejo/cjm039

PMid:17702797

12. Lindaner SJ, Shoff RC. Comparison of essix and havley retainers. J Clin Orthod. 1998;32:95-7.

13. Campbell AM, McMullan RE. The effectiveness of Hawley and vacuum formed retainers: A single-centre randomized controlled trial. AJO. 2007;132:730-7.

14. Sun J, Yn YC, Lin MY, Chen L, Li HW, Zhang L, et al. Survival time comparison between Hawley and clear everlay retainers: A randomized trial. J Dent Res. 2011;90(10):1197-201. https:// doi.org/10.1177/0022034511415274
PMid:21771797

15. Rinchuse $D$, Miles $P$, Shendau J. Orthodontic retention and stability: A clinical perspective. J Clin Orthod. 2007;41(3):125-32. PMid: 17473411

16. Jin C, Bennani F, Gray A, Farella M, Mei L. Survival analysis of orthodontic retainers. Eur J Orthod. 2018;40(5):531-6. https:// doi.org/10.1093/ejo/cjx100 PMid:29370399

17. Hostettler J, Hostettler J. U.S. PatentApplication No. 16/462,232. United States Patent and Trademark Office (USPTO): 2019.

18. Available form: https://www.dental.formlabs.com/ materials/\#dental-It-clear [Last accessed on $2021 \mathrm{Jul} 29]$.

19. Cole, D Evaluation of Fit for 3D-printed Retainers Compared with Thermoform Retainers. Disser; 2018. Available from: https://www.scholarscompass.vcu.edu/etd/5332 [Last accessed on $2021 \mathrm{Jul} 29]$. 